\title{
The Family of Exponential Attractors and Inertial Manifolds for a Generalized Nonlinear Kirchhoff Equations
}

\author{
Guoguang Lin*, Xiaomei Liu \\ Department of Mathematics, Yunnan University, Kunming, China \\ Email: ^gglin@ynu.edu.cn
}

How to cite this paper: Lin, G.G. and Liu, X.M. (2022) The Family of Exponential Attractors and Inertial Manifolds for a Generalized Nonlinear Kirchhoff Equations. Journal of Applied Mathematics and Physics, 10, 172-189.

https://doi.org/10.4236/jamp.2022.101013

Received: November 27, 2021

Accepted: January 21, 2022

Published: January 24, 2022

Copyright $\odot 2022$ by author(s) and Scientific Research Publishing Inc. This work is licensed under the Creative Commons Attribution International License (CC BY 4.0).

http://creativecommons.org/licenses/by/4.0/

\begin{abstract}
In this paper, we study the long-time behavior of a class of generalized nonlinear Kichhoff equation under the condition of $n$ dimension. Firstly, the Lipschitz property and squeezing property of the nonlinear semigroup related to the initial-boundary value problem are proved, and then the existence of its exponential attractor is obtained. By extending the space $E_{0}$ to $E_{k}$, a family of the exponential attractors of the initial-boundary value problem is obtained. In the second part, we consider the long-time behavior for a system of generalized Kirchhoff type with strong damping terms. Using the Hadamard graph transformation method, we obtain the existence of a family of the inertial manifolds while such equations satisfy the spectrum interval condition.
\end{abstract}

\section{Keywords}

A Family of the Exponential Attractors, Inertial Fractal Set, Squeezing

Property, Spectral Gap Condition, A Family of the Inertial Manifolds

\section{Introduction}

Exponential attractor is a compact positive invariant set with finite fractal dimension and exponentially attracts every orbit, which is an important feature to describe the long-term behavior of nonlinear partial differential equations. In reference [1], since Foias and others put forward this concept in 1994, many mathematicians have made in-depth research on exponential attractors. Inertial manifold refers to the positive invariant Lipschitz manifold of finite dimension, which includes the global attractor attracting all solution orbits at exponential speed, and it is an important bridge between infinite dimensional dynamical system and finite dimensional dynamical system. 
In reference [2], the author studied the exponential attractors of the following nonlinear wave equations by using operator decomposition and finite covering methods.

$$
\left\{\begin{array}{l}
u_{t t}+\alpha u_{t}-\Delta u+g(u)=f(x),(x, t) \in \Omega \times R^{+} \\
u=0,(x, t) \in \partial \Omega \times R^{+} \\
u(x, 0)=u_{0}(x), u_{1}(x, 0)=u_{1}(x) .
\end{array}\right.
$$

Contrary to the global attractor, the exponential attractor has a uniform exponential convergence rate on the invariant absorption set of its solution. Because of this, the exponential attractor has deeper and more practical properties, and under the perturbation and numerical approximation, the exponential attractor is more robust than the whole attractor.

In reference [3], Perikles G. Papadopoulos, Nikos M. Stavrakakis studied the global existence and blow-up of the following equations

$$
u_{t t}-\phi(x)\|\nabla u(t)\|^{2} \Delta u+\delta u_{t}=|u|^{\alpha} u, x \in R^{N}, t \geq 0
$$

Initial condition $u(x, 0)=u_{0}(x), u_{t}(x, 0)=u_{1}(x)$.

Li et al. [4]. studied the global existence and blow-up of solutions for the following high-order Kirchhoff type equations with nonlinear dissipation terms

$$
\left\{\begin{array}{l}
u_{t t}+\left(\int_{\Omega}|\nabla u|^{2} \mathrm{~d} x\right)^{q}(-\Delta)^{m} u+u_{t}\left|u_{t}\right|^{r}=|u|^{p} u, x \in \Omega, t>0 \\
\left.u\right|_{\partial \Omega}=0,\left.\frac{\partial^{i} u}{\partial v^{i}}\right|_{\partial \Omega}=0, i=1, \cdots, m-1, t>0 \\
u(x, 0)=u_{0}(x), u_{t}(x, 0)=u_{1}(x), x \in \Omega .
\end{array}\right.
$$

where $\Omega \subset R^{N}(N \geq 1)$ is a bounded open region with smooth boundary, $v$ is an outward normal vector, $m>1$ is a positive integer and $p, q, r>0$ is a normal number. In this paper, using the concavity method, it is obtained that the solution has global existence when $p \leq r$, but when $p>\max \{r, 2 q\}$, for any initial value with negative initial energy, the solution explodes in a finite time with the norm in $L^{p+2}$. Salim [5] not only improves the results in reference [4] by modifying the proof method, but also proves that when the positive initial energy has an upper bound, the solution explodes in a finite time. Inspired by reference [4] [5], Ye et al. [6] studied the following hyperbolic equations of Kirchhoff type with damping term and source term:

$$
\left\{\begin{array}{l}
u_{t t}+\left\|A^{\frac{1}{2}} u\right\|^{2 q} A u+a\left|u_{t}\right|^{q-2} u_{t}=b|u|^{r-2} u, x \in \Omega, t>0 \\
\left.u\right|_{\partial \Omega}=0,\left.\frac{\partial^{i} u}{\partial v^{i}}\right|_{\partial \Omega}=0, i=1, \cdots, m-1, t>0 \\
u(x, 0)=u_{0}(x), u_{t}(x, 0)=u_{1}(x), x \in \Omega .
\end{array}\right.
$$

where $A=(-\Delta)^{m}, m>1$ is a positive integer, $\Omega \subset R^{N}$ is a bounded region 
with smooth boundary, $v$ is an outward normal vector, and $a, b, p>0$ and $q, r>2$ are normal numbers. The author not only obtains the global existence of the solution by constructing a stable set in $H_{0}^{m}$, but also proves the estimation of energy attenuation by using Komornik lemma.

For more research on exponential attractors and inertial manifolds, we can read the literature [7]-[16].

Inspired by the above research, this paper will discuss a family of the existence of exponential attractors and inertial manifolds of a generalized Kirchhoff equation with damping term:

$$
\left\{\begin{array}{l}
u_{t t}+M\left(\left\|\nabla^{m} u\right\|_{p}^{p}\right)(-\Delta)^{2 m} u+\beta(-\Delta)^{2 m} u_{t}+|u|^{\rho}\left(u_{t}+u\right)=f(x), \\
u(x, t)=0, \frac{\partial^{i} u}{\partial v^{i}}=0, i=1,2, \cdots, 2 m-1, x \in \partial \Omega, t>0, \\
u(x, 0)=u_{0}(x), u_{t}(x, 0)=u_{t}(x), x \in \Omega \subset R^{n},
\end{array}\right.
$$

where $m \in N^{+}, \Omega \subset R^{n}(n \geq 1)$ is a bounded domain with smooth boundary $\partial \Omega, f(x)$ is an external force term, $M\left(\left\|\nabla^{m} u\right\|_{p}^{p}\right)$ is the stress term of Kirchhoff equation, $\beta>0, \beta(-\Delta)^{2 m} u_{t}$ is a strong dissipative term, $|u|^{\rho}\left(u_{t}+u\right)$ is a nonlinear source term.

In this paper, our main difficulty is the handling of $M\left(\left\|\nabla^{m} u\right\|_{p}^{p}\right)$ and nonlinear terms $|u|^{\rho}\left(u_{t}+u\right)$. In order to overcome the difficulties, certain assumptions are needed to solve them. The algorithm of proof process has been used by predecessors. The previous algorithms are combined and extended to solve the difficulty of nonlinear term in the paper. This paper is organized as follows. Section 2 is some basic assumptions. Section 3 proves the existence a family of exponential attractors. Section 4 proves the existence of a family of the inertial manifolds by using the Hadamard graph transformation method.

\section{Preliminaries}

For brevity, we used the follow abbreviation:

$$
\begin{aligned}
& \quad H=L^{2}(\Omega),\|\cdot\|_{L^{2}(\Omega)}=\|\cdot\|, H_{0}^{2 m}(\Omega)=H^{2 m}(\Omega) \cap H_{0}^{1}(\Omega), \\
& H_{0}^{2 m+k}(\Omega)=H^{2 m+k}(\Omega) \cap H_{0}^{1}(\Omega), \quad E_{k}=H_{0}^{2 m+k}(\Omega) \times H_{0}^{k}(\Omega),(k=0,1,2, \cdots, 2 m) \\
& \text { and } C_{i}(i=0,1,2, \cdots) \text { denotes positive constant, } \lambda_{1} \text { is the first eigenvalue of } \\
& -\Delta \text { with homogeneous Dirichlet boundary condition on } \Omega \text {. }
\end{aligned}
$$

The notation $(\cdot, \cdot),\|\cdot\|$ for the $H$ inner product and norm,that is $(u, v)=\int u(x) v(x) \mathrm{d} x, \quad(u, u)=\|u\|^{2}$.

(H1) å̃ssume that Kirchhoff type stress term $M(s) \in C^{2}([0,+\infty], R)$ satisfies:

$$
1<\mu_{0} \leq M(s) \leq \mu_{1}, \mu=\left\{\begin{array}{l}
\mu_{0}, \frac{\mathrm{d}}{\mathrm{d} t}\left\|\nabla^{2 m} u\right\|^{2} \geq 0, \\
\mu_{1}, \frac{\mathrm{d}}{\mathrm{d} t}\left\|\nabla^{2 m} u\right\|^{2}<0 .
\end{array}\right.
$$

where $\mu$ is a constant. 
(H2) $\rho \leq \frac{8 m}{n}$.

\section{Exponential Attractors}

We denote the inner product and norm in $E_{k}$ as following:

$$
\forall U_{i}=\left(u_{i}, v_{i}\right) \in E_{k},(i=1,2) \text {, }
$$

we have

$$
\begin{gathered}
\left(U_{1}, U_{2}\right)_{E_{k}}=\left(\nabla^{2 m+k} u_{1}, \nabla^{2 m+k} u_{2}\right)+\left(\nabla^{k} v_{1}, \nabla^{k} v_{2}\right), \\
\|U\|_{E_{k}}^{2}=(U, U)_{E_{k}}=\left\|\nabla^{2 m+k} u\right\|^{2}+\left\|\nabla^{k} v\right\|^{2} .
\end{gathered}
$$

Setting $\forall U=(u, v)^{\mathrm{T}} \in E_{k}, v=u_{t}+\varepsilon u$, then Equation (1.1) can be converted into the following first-order evolution equation

$$
U_{t}+H(U)=F(U)
$$

where

$$
\begin{gathered}
H(U)=\left(\begin{array}{c}
\varepsilon u-v \\
-\varepsilon v+\varepsilon^{2} u+\beta(-\Delta)^{2 m} v+(1-\beta \varepsilon)(-\Delta)^{2 m} u
\end{array}\right) \\
F(U)=\left(\begin{array}{c}
0 \\
{\left[1-M\left(\|\left.\nabla^{m} u\right|_{p} ^{p}\right)\right](-\Delta)^{2 m} u-\left[|u|^{\rho}\left(u_{t}+u\right)\right]+f(x)}
\end{array}\right)
\end{gathered}
$$

In order to accomplish the proof, we need to construct a map. Let $E_{0}, E_{k}$ are two Hilbert spaces with $E_{k} \rightarrow E_{0}$ is dense and continuous injection, and $E_{k} \rightarrow E_{0}$ is compact. Let $S(t)$ is a solution semigroup generated by Equation (3.3).

In the following definitions, $k=1,2, \cdots, 2 m$.

Definition 3.1 [17] $A_{k}$ compact set $M_{k} \subset E_{k}$ is called an exponential attractor for $\left(S(t), B_{k}\right)$ if $A_{k} \subseteq M_{k} \subseteq B_{k}$ and

1) $S(t) M_{k} \subseteq M_{k}, \forall t \geq 0$,

2) $M_{k}$ has finite fractal dimension, $d_{F}\left(M_{k}\right)<+\infty$,

3) There exist universal constans $C_{0}, C_{1}$ such that

$$
\operatorname{dist}\left(S(t) B_{k}, M_{k}\right) \leq C_{0} \mathrm{e}^{-C_{1} t}, \forall t>0,
$$

where $\operatorname{dist}_{E_{k}}\left(A_{k}, B_{k}\right)=\sup _{x \in A_{k}} \inf _{y \in B_{k}}\|x-y\|_{E_{k}}, \quad B_{k}$ is a positively invariant set for $S(t)$ in $E_{k}$.

Definition 3.2 [17] If for every $\delta \in\left(0, \frac{1}{8}\right)$, there exist a time $t^{*}>0$, an integer $N_{0} \geq 1$, and an orthogonal projection $P_{N_{0}}$ of rank equal to $N_{0}$ such that for every $U$ and $V$ in $B_{k}$, either

$$
\left\|S\left(t^{*}\right) U-S\left(t^{*}\right) V\right\|_{E_{k}} \leq \delta\|U-V\|_{E_{k}}
$$

or

$$
\left\|Q_{N_{0}}\left(S\left(t^{*}\right) U-S\left(t^{*}\right) V\right)\right\|_{E_{k}} \leq\left\|P_{N_{0}}\left(S\left(t^{*}\right) U-S\left(t^{*}\right) V\right)\right\|_{E_{k}},
$$


then we call $S(t)$ is squeezing in $B_{k}$, where $Q_{N_{0}}=I-P_{N_{0}}$.

Theorem 3.1 [1] Assume that

1) $S(t)$ possesses a family of $\left(E_{k}, E_{0}\right)$-compact attractors $A_{k}$,

2) $S(t)$ exists a positive invariant compact set $B_{k} \subset E_{0}$,

3) $S(t)$ is a Lipschitz continuous map with a Lipschitz continuous function

$l(t)$ on $B_{k}$, such that $\|S(t) u-S(t) v\|_{E_{k}} \leq l(t)\|u-v\|_{E_{k}}$, and satisfied the discrete squeezing property on $B_{k}$.

Then $S(t)$ has a family of $\left(E_{k}, E_{0}\right)$-compact exponential attractors $M_{k}$ and

$$
M_{k}=\bigcup_{0 \leq t \leq t^{*}} S(t) M_{k}^{*}
$$

where

$$
M_{k}^{*}=A_{k} \cup\left(\bigcup_{j=1}^{\infty} \bigcup_{i=1}^{\infty} S\left(t^{*}\right)^{j}\left(E^{(i)}\right)\right) .
$$

Moreover, the fractal dimension of $M_{k}$ satisfies $d_{F}\left(M_{k}\right) \leq 1+c N_{0}$, where $N_{0}$ is the smallest $N$ which make the discrete squeezing property established, $k=1,2, \cdots, 2 m$.

Proposition $3.1[1]$ There exist $t_{0}\left(D_{k}\right)$ such that

$$
B_{k}=\overline{\bigcup_{0 \leq t \leq t_{0}\left(D_{k}\right)} S(t) D_{k}} \text {. }
$$

is the positive invariant set of $S(t)$ in $E_{0}$, and $B_{k}$ attracts all bounded subsets of $E_{k}$, where $B_{k}$ is a closed bounded absorbing set for $S(t)$ in $E_{k}$.

Proposition 3.2 [1] Let $B_{0}, B_{k}$ respectively are closed bounded absorbing set of Equation (3.3) in $E_{0}, E_{k}$, then $S(t)$ possesses a family of $\left(E_{k}, E_{0}\right)$ -compact attractors $A_{k}$.

Under of the appropriate hypothesized, the initial boundary value problem Equation (1.1) exists unique smooth. This solution possesses the following properties:

$$
\begin{gathered}
\|U\|_{E_{0}}^{2}=\left\|\nabla^{2 m} u\right\|^{2}+\|v\|^{2} \leq C\left(R_{1}\right) \\
\|U\|_{E_{k}}^{2}=\left\|\nabla^{2 m+k} u\right\|^{2}+\left\|\nabla^{k} v\right\|^{2} \leq C\left(R_{2}\right) .
\end{gathered}
$$

We denote the solution in Theorem 3.1 by $S(t)\left(U_{0}\right)=U$, the $S(t)$ is a continuous semigroup in $E_{0}$, There exist the balls:

$$
\begin{aligned}
& D_{0}=\left\{U \in E_{0}:\|U\|_{E_{0}}^{2} \leq C\left(R_{1}\right)\right\}, \\
& D_{k}=\left\{U \in E_{k}:\|U\|_{E_{k}}^{2} \leq C\left(R_{2}\right)\right\},
\end{aligned}
$$

respectively is a absorbing set of $S(t)$ in $E_{0}$ and $E_{k}$.

Lemma 3.1 For $\forall U=(u, v)^{\mathrm{T}} \in E_{k}$, when we can obtain

$$
(H(U), U)_{E_{k}} \geq k_{1}\|U\|_{E_{k}}^{2}+k_{2}\left\|\nabla^{2 m+k} v\right\|^{2} .
$$


Proof. By (3.1), (3.4) we get

$$
\begin{aligned}
& (H(U), U)_{E_{k}} \\
= & \left(\varepsilon\left(\nabla^{2 m+k} u\right)-\nabla^{2 m+k} v, \nabla^{2 m+k} u\right) \\
& +\left(-\varepsilon \nabla^{k} v+\varepsilon^{2} \nabla^{k} u+\beta(-\Delta)^{2 m+\frac{k}{2}} v+(1-\beta \varepsilon)(-\Delta)^{2 m+\frac{k}{2}} u, \nabla^{k} v\right) \\
= & \varepsilon\left\|\nabla^{2 m+k} u\right\|^{2}-\varepsilon\left\|\nabla^{k} v\right\|^{2}+\varepsilon^{2}\left(\nabla^{k} u, \nabla^{k} v\right)+\beta\left\|\nabla^{2 m+k} v\right\|^{2} \\
& -\beta \varepsilon\left(\nabla^{2 m+k} u, \nabla^{2 m+k} v\right) .
\end{aligned}
$$

By employing Hölder's inequality, Young's inequality and Poincaré's inequality, we process the terms in (3.16), we have

$$
\begin{gathered}
\varepsilon^{2}\left(\nabla^{k} u, \nabla^{k} v\right) \geq-\frac{\varepsilon^{2}}{2}\left\|\nabla^{k} u\right\|^{2}-\frac{\varepsilon^{2}}{2}\left\|\nabla^{k} v\right\|^{2} \geq-\frac{\varepsilon^{2}}{2 \lambda_{1}^{2 m}}\left\|\nabla^{2 m+k} u\right\|^{2}-\frac{\varepsilon^{2}}{2}\left\|\nabla^{k} v\right\|^{2} . \\
-\beta \varepsilon\left(\nabla^{2 m+k} u, \nabla^{2 m+k} v\right) \geq-\frac{\beta \varepsilon}{2}\left\|\nabla^{2 m+k} u\right\|^{2}-\frac{\beta \varepsilon}{2}\left\|\nabla^{2 m+k} v\right\|^{2} .
\end{gathered}
$$

By the value of $\varepsilon$, and substituting (3.17)-(3.18), we have

$$
\begin{aligned}
(H(U), U)_{E_{k}} \geq & \left(\varepsilon-\frac{\varepsilon^{2}}{2 \lambda_{1}^{2 m}}-\frac{\beta \varepsilon}{2}\right)\left\|\nabla^{2 m+k} u\right\|^{2} \\
& +\left(\frac{\beta \lambda_{1}^{2 m}}{2}-\varepsilon-\frac{\varepsilon^{2}}{2}\right)\left\|\nabla^{k} v\right\|^{2}+\left(\frac{\beta}{2}-\frac{\beta \varepsilon}{2}\right)\left\|\nabla^{2 m+k} v\right\|^{2} .
\end{aligned}
$$

because of $0<\varepsilon<\min \left\{2 \lambda_{1}^{2 m}-\beta \lambda_{1}^{2 m}, \sqrt{1+\beta \lambda_{1}^{2 m}}-1,1\right\}$, so $\varepsilon-\frac{\varepsilon^{2}}{2 \lambda_{1}^{2 m}}-\frac{\beta \varepsilon}{2} \geq 0$, $\frac{\beta \lambda_{1}^{2 m}}{2}-\varepsilon-\frac{\varepsilon^{2}}{2} \geq 0, \frac{\beta}{2}-\frac{\beta \varepsilon}{2} \geq 0$.

Let $k_{1}=\min \left\{\varepsilon-\frac{\varepsilon^{2}}{2 \lambda_{1}^{2 m}}-\frac{\beta \varepsilon}{2}, \frac{\beta \lambda_{1}^{2 m}}{2}-\varepsilon-\frac{\varepsilon^{2}}{2}\right\}, k_{2}=\frac{\beta}{2}-\frac{\beta \varepsilon}{2}$, we can get

$$
(H(U), U)_{E_{k}} \geq k_{1}\|U\|_{E_{k}}^{2}+k_{2}\left\|\nabla^{2 m+k} v\right\|^{2}
$$

The proof is completed.

Let $S(t) U_{0}=U(t)=(u(t), v(t))^{\mathrm{T}}$, where $v(t)=u_{t}(t)+\varepsilon u(t)$,

$S(t) V_{0}=V(t)=(\bar{u}(t), \bar{v}(t))^{\mathrm{T}}$, where $\bar{v}(t)=\bar{u}_{t}(t)+\varepsilon \bar{u}(t)$,

Next set $\phi(t)=S(t) U_{0}-S(t) V_{0}=U(t)-V(t)=(w(t), z(t))^{\mathrm{T}}$, where $z(t)=w_{t}(t)+\varepsilon w(t)$, then $\phi(t)$ satisfies:

$$
\begin{gathered}
\phi_{t}(t)+H(U)-H(V)+F(V)-F(U)=0, \\
\phi_{0}(0)=U_{0}-V_{0} .
\end{gathered}
$$

In order to certify Equation (1.1) exists a family of exponential attractors,we first show the semigroup $S(t)$ of system (1.1) is Lipschitz continuous on $B_{k}$.

Lemma 3.2 (Lipschitz property) For $\forall U_{0}, V_{0} \in B_{k}$, where $U_{0}, V_{0}$ is the initial values of problem (1.1), and $t \geq 0$, we have 


$$
\left\|S(t) U_{0}-S(t) V_{0}\right\|_{E_{k}}^{2} \leq \mathrm{e}^{r t}\left\|U_{0}-V_{0}\right\|_{E_{k}}^{2} .
$$

Proof. Taking the inner product of the Equation (3.20) with $\phi(t)$ in $E_{k}$, we have

$$
\begin{aligned}
& \frac{1}{2} \frac{\mathrm{d}}{\mathrm{d} t}\|\phi(t)\|_{E_{k}}^{2}+(H(U)-H(V), \phi(t))_{E_{k}}-\left((-\Delta)^{2 m+\frac{k}{2}} w, \nabla^{k} z(t)\right) \\
& +\left(M\left(\|\left.\nabla^{m} u\right|_{p} ^{p}\right)(-\Delta)^{2 m+\frac{k}{2}} u-M\left(\left\|\nabla^{m} \bar{u}\right\|_{p}^{p}\right)(-\Delta)^{2 m+\frac{k}{2}} \bar{u}, \nabla^{k} z(t)\right) \\
& +\left(\nabla^{k}\left(|u|^{\rho}\left(u_{t}+u\right)-|\bar{u}|^{\rho}\left(\bar{u}_{t}+\bar{u}\right)\right), \nabla^{k} z(t)\right)=0 .
\end{aligned}
$$

Next, we deal with the following items one by one. Similar to Lemma 3.1, we easily obtain

$$
(H(U)-H(V), \phi(t))_{E_{k}}=(H(\phi(t)), \phi(t))_{E_{k}} \geq k_{1}\|\phi(t)\|_{E_{k}}^{2}+k_{2}\left\|\nabla^{2 m+k} z(t)\right\|^{2} .
$$

For convenience, let's call $s=\left\|\nabla^{m} u\right\|_{p}^{p}, \bar{s}=\left\|\nabla^{m} \bar{u}\right\|_{p}^{p}$, then by (H1) and using the mean value theorem, Young's inequality, we have

$$
\begin{aligned}
& \left(M\left(\left\|\nabla^{m} \bar{u}\right\|_{p}^{p}\right)(-\Delta)^{2 m+\frac{k}{2}} \bar{u}-M\left(\left\|\nabla^{m} u\right\|_{p}^{p}\right)(-\Delta)^{2 m+\frac{k}{2}} u, \nabla^{k} z(t)\right) \\
& \leq\left|\left(M(\bar{s})(-\Delta)^{2 m+\frac{k}{2}} w, \nabla^{k} z(t)\right)\right|+\left|\left(M^{\prime}(\zeta)(\bar{s}-s)(-\Delta)^{2 m+\frac{k}{2}} u, \nabla^{k} z(t)\right)\right| \\
& \leq \frac{\mu_{1}}{2}\left\|\nabla^{2 m+k} w\right\|^{2}+\frac{\mu_{1}}{2}\left\|\nabla^{2 m+k} z\right\|^{2}+C_{2}\left\|\nabla^{2 m+k} w\right\|\left\|\nabla^{2 m+k} z\right\| \\
& \leq \frac{\mu_{1}+C_{2}}{2}\left\|\nabla^{2 m+k} w\right\|^{2}+\frac{\mu_{1}+C_{2}}{2}\left\|\nabla^{2 m+k} z\right\|^{2}
\end{aligned}
$$

For the last term, we apply the mean value theorem, by (H2), we have

$$
\begin{aligned}
& \left(\nabla^{k}\left(|\bar{u}|^{\rho}\left(\bar{u}_{t}+\bar{u}\right)-|u|^{\rho}\left(u_{t}+u\right)\right), \nabla^{k} z(t)\right) \\
& \leq C_{3} \int\left(|\bar{u}|^{\rho}+|u|^{\rho}\right) \mathrm{d} x\left|\nabla^{k} w_{t}\right|\left|\nabla^{k} z\right|+C_{4} \int\left(|\bar{u}|^{\rho}+|u|^{\rho}\right) \mathrm{d} x\left|\nabla^{k} w\right|\left|\nabla^{k} z\right| \\
& \leq C_{3}\left(\left\|\left.\bar{u}\right|^{\rho}\right\|_{L^{\infty}(\Omega)}+\left\||u|^{\rho}\right\|_{L^{\infty}(\Omega)}\right)\left(\frac{\left\|\nabla^{k} w_{t}\right\|^{2}}{2}+\frac{\left\|\nabla^{k} z\right\|^{2}}{2}\right) \\
& +C_{4}\left(\left\||\bar{u}|^{\rho}\right\|_{L^{\infty}(\Omega)}+\left\|\left.||\right|^{\rho}\right\|_{L^{\infty}(\Omega)}\right)\left(\frac{\left\|\nabla^{k} w\right\|^{2}}{2}+\frac{\left\|\nabla^{k} z\right\|^{2}}{2}\right),
\end{aligned}
$$

By the interpolation inequality

$$
\|\bar{u}\|_{\infty}^{\rho} \leq C_{5}\left\|\nabla^{2 m} \bar{u}\right\|^{\frac{\rho n}{4 m}}
$$

In the same way with

$$
\|u\|_{\infty}^{\rho} \leq C_{6}\left\|\nabla^{2 m} u\right\|^{\frac{\rho n}{4 m}},
$$

where $\rho \leq \frac{8 m}{n}$. 
Therefore

$$
\begin{aligned}
& \left(\nabla^{k}\left(|u|^{\rho}\left(u_{t}+u\right)-|\bar{u}|^{\rho}\left(\bar{u}_{t}+\bar{u}\right)\right), \nabla^{k} z(t)\right) \\
& \leq C_{7}\left(\left\|\nabla^{2 m} u\right\|^{\frac{\rho n}{4 m}}+\left\|\nabla^{2 m} \bar{u}\right\|^{\frac{\rho n}{4 m}}\right)\left\|\nabla^{k} w_{t}\right\|^{2} \\
& +C_{8}\left(\left\|\nabla^{2 m} u\right\|^{\frac{\rho n}{4 m}}+\left\|\nabla^{2 m} \bar{u}\right\|^{\frac{\rho n}{4 m}}\right)\left\|\nabla^{k} z\right\|^{2} \\
& +C_{9}\left(\left\|\nabla^{2 m} u\right\|^{\frac{\rho n}{4 m}}+\left\|\nabla^{2 m} \bar{u}\right\|^{\frac{\rho n}{4 m}}\right)\left\|\nabla^{k} w\right\|^{2} \\
& \leq\left(\lambda_{1}^{2 m} C_{9}\left(\left\|\nabla^{2 m} u\right\|^{\frac{\rho n}{4 m}}+\left\|\nabla^{2 m} \bar{u}\right\| \frac{\rho n}{4 m}\right)\right)\left\|\nabla^{2 m+k} w\right\|^{2} \\
& \quad+C_{8}\left(\left\|\nabla^{2 m} u\right\|^{\frac{\rho n}{4 m}}+\left\|\nabla^{2 m} \bar{u}\right\|^{\frac{\rho n}{4 m}}\right)\left\|\nabla^{k} z\right\|^{2} .
\end{aligned}
$$

Integrating (3.24) - (3.26) into (3.23), we have

$$
\begin{aligned}
& \frac{1}{2} \frac{\mathrm{d}}{\mathrm{d} t}\|\phi(t)\|_{E_{k}}^{2}+k_{1}\|\phi(t)\|^{2}+\left(k_{2}-\frac{\mu_{1}+C_{2}}{2}-1\right)\left\|\nabla^{2 m+k} z\right\|^{2} \\
& \leq\left(\frac{\mu_{1}+C_{2}}{2}+\lambda_{1}^{2 m} C_{9}\left(\left\|\nabla^{2 m} u\right\|^{\frac{\rho n}{4 m}}+\left\|\nabla^{2 m} \bar{u}\right\|^{\frac{\rho n}{4 m}}\right)+1\right)\left\|\nabla^{2 m+k} w\right\|^{2} \\
& \quad+C_{8}\left(\left\|\nabla^{2 m} u\right\|^{\frac{\rho n}{4 m}}+\left\|\nabla^{2 m} \bar{u}\right\|^{\frac{\rho n}{4 m}}\right)\left\|\nabla^{k} z\right\|^{2} \\
& \leq C_{10}\|\phi(t)\|_{E_{k}}^{2} .
\end{aligned}
$$

where

$C_{10}=\max \left\{\frac{\mu_{1}+C_{2}}{2}+\lambda_{1}^{2 m} C_{9}\left(\left\|\nabla^{2 m} u\right\|^{\frac{\rho n}{4 m}}+\left\|\nabla^{2 m} \bar{u}\right\|^{\frac{\rho n}{4 m}}\right)-1, C_{8}\left(\left\|\nabla^{2 m} u\right\|^{\frac{\rho n}{4 m}}+\left\|\nabla^{2 m} \bar{u}\right\|^{\frac{\rho n}{4 m}}\right)\right\}$.

By using Gronwall's inequality, we have

$$
\|\phi(t)\|_{E_{k}}^{2} \leq \mathrm{e}^{2 C_{10} t}\|\phi(0)\|_{E_{k}}^{2}=\mathrm{e}^{r t}\|\phi(0)\|_{E_{k}}^{2} .
$$

where $r=2 C_{10}$, so we have

$$
\left\|S(t) U_{0}-S(t) V_{0}\right\|_{E_{k}}^{2} \leq \mathrm{e}^{r t}\left\|U_{0}-V_{0}\right\|_{E_{k}}^{2} .
$$

The proved is completed.

Now,we introduce the operator $-\Delta$, Obviously, $-\Delta$ is an unbounded self-adjoin positive operator and $(-\Delta)^{-1}$ is compact. So, there is an orthonormal basis $\left\{w_{i}\right\}_{i=1}^{\infty}$ of $H$ consisting of eigenvectors $w_{j}$ of $-\Delta$ such that $(-\Delta) w_{j}=\lambda_{j} w_{j}, \quad 0<\lambda_{1} \leq \lambda_{2} \leq \cdots \leq \lambda_{j} \rightarrow+\infty . \forall N$ denote by $P=P_{N}$ the projector, $P_{N}$ is an orthogonal projection, $Q=Q_{N}=I-P_{N}$.

As follows,we will need

$$
\left\|(-\Delta)^{2 m} u\right\| \geq \lambda_{N+1}^{2 m}\|u\|, u \in Q_{N}\left(H^{2 m}(\Omega) \cap H_{0}^{1}(\Omega)\right),
$$

Lemma 3.3 For $\forall U_{0}, V_{0} \in B_{k}$, where $U_{0}, V_{0}$ is the initial values of problem 
(1.1). Let

$$
Q_{n_{0}}(t)=Q_{n_{0}}(U(t)-V(t))=Q_{n_{0}}(\phi(t))=\left(w_{n_{0}}(t), z_{n_{0}}(t)\right)^{\mathrm{T}}
$$

then we have

$$
\left\|\phi_{n_{0}}(t)\right\|_{E_{k}}^{2} \leq\left(\mathrm{e}^{-2 k_{1} t}+\frac{C_{19} \lambda_{N+1}^{2 m}}{2 k_{1}+r} \mathrm{e}^{r t}\right)\left\|\phi_{n_{0}}(0)\right\|_{E_{k}}^{2} .
$$

Proof. Applying $Q_{n_{0}}$ to (3.20), we have

$$
\phi_{n_{0} t}(t)+Q_{n_{0}}(H(U)-H(V))+Q_{n_{0}}(F(V)-F(U))=0 .
$$

Taking the inner product of (3.31) with $Q_{n_{0}}(t)$ in $E_{k}$, we have

$$
\begin{aligned}
& \frac{1}{2} \frac{\mathrm{d}}{\mathrm{d} t}\left\|\phi_{n_{0}}(t)\right\|_{E_{k}}^{2}+k_{1}\left\|\phi_{n_{0}}(t)\right\|_{E_{k}}^{2}+k_{2}\left(\left\|\nabla^{2 m+k} z_{n_{0}}(t)\right\|^{2}\right) \\
& -\left((-\Delta)^{2 m+\frac{k}{2}} w_{n_{0}}(t), \nabla^{k} z_{n_{0}}(t)\right) \\
& +\left(Q_{n_{0}}\left(M\left(\left\|\nabla^{m} u\right\|_{p}^{p}\right)(-\Delta)^{2 m+\frac{k}{2}} u-M\left(\left\|\nabla^{m} \bar{u}\right\|_{p}^{p}\right)(-\Delta)^{2 m+\frac{k}{2}} \bar{u}\right), \nabla^{k} z_{n_{0}}(t)\right) \\
& +\left(\nabla^{k}\left(Q_{n_{0}}\left(|u|^{\rho}\left(u_{t}+u\right)-\mid \bar{u}^{\rho}\left(\bar{u}_{t}+\bar{u}\right)\right)\right), \nabla^{k} z_{n_{0}}(t)\right)=0 .
\end{aligned}
$$

Next, we deal with the following items one by one

$$
\begin{aligned}
& \left(Q_{n_{0}}\left(M\left(\left\|\nabla^{m} \bar{u}\right\|_{p}^{p}\right)(-\Delta)^{2 m+\frac{k}{2}} \bar{u}-M\left(\left\|\nabla^{m} u\right\|_{p}^{p}\right)(-\Delta)^{2 m+\frac{k}{2}} u\right), \nabla^{k} z_{n_{0}}(t)\right) \\
& =\left(M\left(\bar{s}_{n_{0}}\right)(-\Delta)^{2 m+\frac{k}{2}} \bar{u}_{n_{0}}-M\left(s_{n_{0}}\right)(-\Delta)^{2 m+\frac{k}{2}} u_{n_{0}}, \nabla^{k} z_{n_{0}}(t)\right) \\
& \leq\left|\left(M\left(\bar{s}_{n_{0}}\right)(-\Delta)^{2 m+\frac{k}{2}} w_{n_{0}}, \nabla^{k} z_{n_{0}}(t)\right)\right| \\
& +\left|\left(M^{\prime}(\zeta)\left(\bar{s}_{n_{0}}-s_{n_{0}}\right)(-\Delta)^{2 m+\frac{k}{2}} u_{n_{0}}, \nabla^{k} z_{n_{0}}(t)\right)\right| \\
& \leq \frac{\mu_{1}}{2}\left\|\nabla^{2 m+k} w_{n_{0}}\right\|^{2}+\frac{\mu_{1}}{2}\left\|\nabla^{2 m+k} z_{n_{0}}\right\|^{2}+C_{11}\left\|\nabla^{2 m+k} w_{n_{0}}\right\| \nabla^{2 m+k} z_{n_{0}} \| \\
& \leq\left(\frac{\mu_{1}+C_{11}}{2}\right)\left\|\nabla^{2 m+k} w_{n_{0}}\right\|^{2}+\left(\frac{\mu_{1}+C_{11}}{2}\right)\left\|\nabla^{2 m+k} z_{n_{0}}\right\|^{2} .
\end{aligned}
$$

For the last term, we apply the mean value theorem, by $(\mathrm{H} 2)$, we have

$$
\begin{aligned}
& \left(\nabla^{k}\left(Q_{n_{0}}\left(|\bar{u}|^{\rho}\left(\bar{u}_{t}+\bar{u}\right)\right)-|u|^{\rho}\left(u_{t}+u\right)\right), \nabla^{k} z_{n_{0}}(t)\right) \\
& =\left(\nabla^{k}\left(\left|\bar{u}_{n_{0}}\right|^{\rho}\left(\bar{u}_{n_{0} t}+\bar{u}_{n_{0}}\right)-\left|u_{n_{0}}\right|^{\rho}\left(u_{n_{0}}+u_{n_{0}}\right)\right), \nabla^{k} z_{n_{0}}(t)\right) \\
& \leq C_{12}\left(\left\|\left|\bar{u}_{n_{0}}\right|^{\rho}\right\|_{L^{\infty}(\Omega)}+\left\|\left.|| u_{n_{0}}\right|^{\rho}\right\|_{L^{\infty}(\Omega)}\right)\left(\frac{\left\|\nabla^{k} w_{n_{0} t}\right\|^{2}}{2}+\frac{\left\|\nabla^{k} z_{n_{0}}\right\|^{2}}{2}\right) \\
& +C_{13}\left(\left\|\left.\bar{u}_{n_{0}}\right|^{\rho}\right\|_{L^{\infty}(\Omega)}+\left\|\left|u_{n_{0}}\right|^{\rho}\right\|_{L^{\infty}(\Omega)}\right)\left(\frac{\left\|\nabla^{k} w_{n_{0}}\right\|^{2}}{2}+\frac{\|\left.\nabla^{k} z_{n_{0}}\right|^{2}}{2}\right)
\end{aligned}
$$


By the interpolation inequality

$$
\left\|\bar{u}_{n_{0}}\right\|_{\infty}^{\rho} \leq C_{14}\left\|\nabla^{2 m} \bar{u}_{n_{0}}\right\|^{\frac{\rho n}{4 m}},
$$

In the same way with

$$
\left\|u_{n_{0}}\right\|_{\infty}^{\rho} \leq C_{15}\left\|\nabla^{2 m} u_{n_{0}}\right\|^{\frac{\rho n}{4 m}},
$$

where $\rho \leq \frac{8 m}{n}$.

Therefore

$$
\begin{aligned}
& \left(\nabla^{k}\left(Q_{n_{0}}\left(|\bar{u}|^{\rho}\left(\bar{u}_{t}+\bar{u}\right)\right)-|u|^{\rho}\left(u_{t}+u\right)\right), \nabla^{k} z_{n_{0}}(t)\right) \\
& \leq C_{16}\left(\left\|\nabla^{2 m} u_{n_{0}}\right\|^{\frac{\rho n}{4 m}}+\left\|\nabla^{2 m} \bar{u}_{n_{0}}\right\| \frac{\rho n}{4 m}\right)\left\|\nabla^{k} w_{n_{0} t}\right\|^{2} \\
& +C_{17}\left(\left\|\nabla^{2 m} u_{n_{0}}\right\|^{\frac{\rho n}{4 m}}+\left\|\nabla^{2 m} \bar{u}_{n_{0}}\right\| \frac{\rho n}{4 m}\right)\left\|\nabla^{k} z_{n_{0}}\right\|^{2} \\
& +C_{18}\left(\left\|\nabla^{2 m} u_{n_{0}}\right\|^{\frac{\rho n}{4 m}}+\left\|\nabla^{2 m} \bar{u}_{n_{0}}\right\| \|^{\frac{\rho n}{4 m}}\right)\left\|\nabla^{k} w_{n_{0}}\right\|^{2} \\
& \leq\left(\lambda_{N+1}^{2 m} C_{18}\left(\left\|\nabla^{2 m} u_{n_{0}}\right\|^{\frac{\rho n}{4 m}}+\left\|\nabla^{2 m} \bar{u}_{n_{0}}\right\| \frac{\rho n}{4 m}\right)\right)\left\|\nabla^{2 m+k} w_{n_{0}}\right\|^{2} \\
& +C_{17}\left(\left\|\nabla^{2 m} u_{n_{0}}\right\|^{\frac{\rho n}{4 m}}+\left\|\nabla^{2 m} \bar{u}_{n_{0}}\right\| \frac{\rho n}{4 m}\right)\left\|\nabla^{k} z_{n_{0}}\right\|^{2}
\end{aligned}
$$

Integrating (3.33) - (3.34) into (3.32), we have

$$
\begin{aligned}
& \frac{1}{2} \frac{\mathrm{d}}{\mathrm{d} t}\left\|\phi_{n_{0}}(t)\right\|_{E_{k}}^{2}+k_{1}\left\|\phi_{n_{0}}(t)\right\|^{2}+\left(k_{2}-\frac{\mu_{1}+C_{11}}{2}-1\right)\left\|\nabla^{2 m+k} z_{n_{0}}\right\|^{2} \\
& \leq\left(\frac{\mu_{1}+C_{11}}{2}+\lambda_{N+1}^{2 m} C_{18}\left(\left\|\nabla^{2 m} u_{n_{0}}\right\| \frac{\rho n}{4 m}+\left\|\nabla^{2 m} \bar{u}_{n_{0}}\right\| \frac{\rho n}{4 m}\right)+1\right)\left\|\nabla^{2 m+k} w_{n_{0}}\right\|^{2} \\
& \quad+C_{17}\left(\left\|\nabla^{2 m} u_{n_{0}}\right\|^{\frac{\rho n}{4 m}}+\left\|\nabla^{2 m} \bar{u}_{n_{0}}\right\| \|^{\frac{\rho n}{4 m}}\right)\left\|\nabla^{k} z_{n_{0}}\right\|^{2} \\
& \leq C_{19} \lambda_{N+1}^{2 m}\left\|\phi_{n_{0}}(t)\right\|_{E_{k}}^{2} .
\end{aligned}
$$

where

$$
C_{19}=\max \left\{\left(\frac{\mu_{1}+C_{11}+2}{2 \lambda_{N+1}^{2 m}}+C_{18}\left(\left\|\nabla^{2 m} u_{n_{0}}\right\|^{\frac{\rho n}{4 m}}+\left\|\nabla^{2 m} \bar{u}_{n_{0}}\right\| \frac{\rho n}{4 m}\right)\right), \frac{C_{17}\left(\left\|\nabla^{2 m} u_{n_{0}}\right\|^{\frac{\rho n}{4 m}}+\left\|\nabla^{2 m} \bar{u}_{n_{0}}\right\|^{\frac{\rho n}{4 m}}\right)}{\lambda_{N+1}^{2 m}}\right\} .
$$

Using Gronwall's inequality, we have

$$
\left\|\phi_{n_{0}}(t)\right\|_{E_{k}}^{2} \leq\left(\mathrm{e}^{-2 k_{1} t}+\frac{C_{19} \lambda_{N+1}^{2 m}}{2 k_{1}+r} \mathrm{e}^{r t}\right)\left\|\phi_{n_{0}}(0)\right\|_{E_{k}}^{2} .
$$

The proved is completed. 
Lemma 3.4 (squeezing property) For $\forall U_{0}, V_{0} \in B_{k}$, if

$$
\left\|P_{n_{0}}\left(S\left(t^{*}\right) U_{0}-S\left(t^{*}\right) V_{0}\right)\right\|_{E_{k}} \leq\left\|\left(I-P_{n_{0}}\right)\left(S\left(t^{*}\right) U_{0}-S\left(t^{*}\right) V_{0}\right)\right\|_{E_{k}},
$$

then we have

$$
\left\|S\left(t^{*}\right) U_{0}-S\left(t^{*}\right) V_{0}\right\|_{E_{k}} \leq \frac{1}{8}\left\|U_{0}-V_{0}\right\|_{E_{k}} .
$$

Proof. If $\left\|P_{n_{0}}\left(S\left(t^{*}\right) U_{0}-S\left(t^{*}\right) V_{0}\right)\right\|_{E_{k}} \leq\left\|\left(I-P_{n_{0}}\right)\left(S\left(t^{*}\right) U_{0}-S\left(t^{*}\right) V_{0}\right)\right\|_{E_{k}}$, then

$$
\begin{aligned}
& \left\|S\left(t^{*}\right) U_{0}-S\left(t^{*}\right) V_{0}\right\|_{E_{k}}^{2} \\
& \leq\left\|P_{n_{0}}\left(S\left(t^{*}\right) U_{0}-S\left(t^{*}\right) V_{0}\right)\right\|_{E_{k}}^{2}+\left\|\left(I-P_{n_{0}}\right)\left(S\left(t^{*}\right) U_{0}-S\left(t^{*}\right) V_{0}\right)\right\|_{E_{k}}^{2} \\
& \leq 2\left\|\left(I-P_{n_{0}}\right)\left(S\left(t^{*}\right) U_{0}-S\left(t^{*}\right) V_{0}\right)\right\|_{E_{k}}^{2} \\
& \leq 2\left(\mathrm{e}^{-2 k_{1} t^{*}}+\frac{C_{19} \lambda_{N+1}^{2 m}}{2 k_{1}+r} \mathrm{e}^{r t^{*}}\right)\left\|U_{0}-V_{0}\right\|_{E_{k}}^{2} .
\end{aligned}
$$

Let $t^{*}$ be large enough

$$
\mathrm{e}^{-2 k_{1}{ }^{*}} \leq \frac{1}{256}
$$

Also let $n_{0}$ be large enough

$$
\frac{C_{19} \lambda_{N+1}^{2 m}}{2 k_{1}+r} \mathrm{e}^{r t^{*}} \leq \frac{1}{256} .
$$

Subsituting (3.39) - (3.41) into (3.38), we have

$$
\left\|S\left(t^{*}\right) U_{0}-S\left(t^{*}\right) V_{0}\right\|_{E_{k}} \leq \frac{1}{8}\left\|U_{0}-V_{0}\right\|_{E_{k}} .
$$

The proved is completed.

Theorem 3.2 Under the above assumptions, $U_{0} \in E_{k}, \quad k=1,2, \cdots, 2 m$, $f \in H$.Then the initial boundary value problem (1.1) the solution semigroup has a family of $\left(E_{k}, E_{0}\right)$-compact exponential attractors $M_{k}$ on $B_{k}$, $M_{k}=\bigcup_{0 \leq t \leq t^{*}} S(t)\left(A_{k} \cup\left(\bigcup_{j=1}^{\infty} \bigcup_{i=1}^{\infty} S\left(t^{*}\right)^{j}\left(E^{(i)}\right)\right)\right)$, and the fractal dimension is satisfied $d_{F}\left(M_{k}\right) \leq 1+c N_{0}$.

Proof. According to Theorem 3.1, Lemma 3.2, Theorem 3.2 is easily proven.

\section{A Family of Inertial Manifolds}

Next, we will prove the existence of a family of inertial manifolds when $N$ is large enough by using graph norm transformation method.

Definition 4.1 [18] Let $S(t)=\{S(t)\}_{t \geq 0}$ be the solution semigroup on Banach space $E_{k}=H_{0}^{2 m+k}(\Omega) \times H_{0}^{k}(\Omega)(k=1,2, \cdots, 2 m)$, and there is a subset $\mu_{k} \subset E_{k}:$

1) $\mu_{k}$ is a finite-dimensional Lipschitz manifold; 
2) $\mu_{k}$ is the positive invariant set, that is $S(t) \mu_{k} \subset \mu_{k}, \forall t>0$;

3) $\mu_{k}$ attracts exponentially all orbits of solutions, that is, there are constants $\eta>0, C>0$, Such that

$$
d\left(S(t) x, \mu_{k}\right) \leq C \mathrm{e}^{-\eta t}, \forall t \geq 0, \forall x \in E_{k} ;
$$

It is said that $\mu_{k}$ is an inertial manifold about $S(t)=\{S(t)\}_{t \geq 0}$.

Definition 4.2 [18] Let the operator $\Lambda: E_{k} \rightarrow E_{k}$ have several eigenvalues of positive real parts, and its eigenfunction $\left\{w_{j}\right\}_{j \geq 1}$ expands into the corresponding orthogonal space in $E_{k}$, and $F \in C_{b}\left(E_{k}, E_{k}\right)$ satisfies the Lipschitz condition

$$
\|F(U)-F(V)\|_{E_{k}} \leq l_{F}\|U-V\|_{E_{k}}, U, V \in E_{k} .
$$

If the point spectrum of the operator can be divided into two parts $\sigma_{1}$ and $\sigma_{2}$, where $\sigma_{1}$ is finite,

$$
\begin{gathered}
\Lambda_{k, 1}=\sup \left\{\operatorname{Re} \lambda \mid \lambda \in \sigma_{1}\right\}, \Lambda_{k, 2}=\sup \left\{\operatorname{Re} \lambda \mid \lambda \in \sigma_{2}\right\}, \\
E_{k_{i}}=\operatorname{span}\left\{w_{j} \mid j \in \sigma_{i}\right\}, i=1,2 .
\end{gathered}
$$

Then

$$
\begin{gathered}
\Lambda_{k, 2}-\Lambda_{k, 1}>4 l_{F}, \\
E_{k}=E_{k_{1}} \oplus E_{k_{2}},
\end{gathered}
$$

hold with continuous orthogonal projection $P_{k, 1}: E_{k} \rightarrow E_{k_{1}}, P_{k, 2}: E_{k} \rightarrow E_{k_{2}}$, So it is said that the operator $\Lambda$ satisfies the spectral interval condition, $P$ is orthogonal projection.

Lemma 4.1 Let the eigenvalues $\mu_{j}^{ \pm}, j \geq 1$ is non-decreasing and for every $n \in N$, when $N \geq n$, such that $\mu_{N}^{-}$and $\mu_{N+1}^{-}$are consecutive adjacent values.

Equation (1.1) are equivalent to the following first-order evolution equation:

$$
U_{t}+\Lambda U=F(U),
$$

with

$$
\begin{aligned}
& U=(u, v)^{\mathrm{T}} \in E_{k}, v=u_{t}, \Lambda=\left(\begin{array}{cc}
0 & -I \\
M\left(\left\|\nabla^{m} u\right\|_{p}^{p}\right)(-\Delta)^{2 m} & \beta(-\Delta)^{2 m}
\end{array}\right), \\
& F(U)=\left(\begin{array}{c}
0 \\
f(x)-|u|^{\rho}\left(u_{t}+u\right)
\end{array}\right) .
\end{aligned}
$$

we consider the graph norm on $E_{k}$, which induced by the scale product

$$
(U, V)_{E_{k}}=\left(M\left(\left\|\nabla^{m} u\right\|_{p}^{p}\right)(-\Delta)^{2 m+k} u,(-\Delta)^{2 m+k} \bar{y}\right)+\left(\nabla^{k} \bar{g}, \nabla^{k} v\right)
$$

where $U=(u, v)^{\mathrm{T}}, V=(y, g)^{\mathrm{T}} \in E_{k} ; \bar{y}, \bar{g}$ represent the conjugation of $y, g$ respectively; $u, v, y, g \in H^{2 m+k}(\Omega)$. Obviously, the operator $\Lambda$ defined in (4.2) is monotone. Indeed, for $U \in E_{k}$, 


$$
\begin{aligned}
(\Lambda U, U)_{E_{k}}= & \left(\left(-v, M\left(\left\|\nabla^{m} u\right\|_{p}^{p}\right)(-\Delta)^{2 m} u+\beta(-\Delta)^{2 m} v\right),(u, v)\right) \\
= & \left(-M\left(\left\|\nabla^{m} u\right\|_{p}^{p}\right)(-\Delta)^{2 m+k} v,(-\Delta)^{2 m+k} \bar{u}\right) \\
& +\left(\nabla^{k} \bar{v}, \nabla^{k}\left(M\left(\left\|\nabla^{m} u\right\|_{p}^{p}\right)(-\Delta)^{2 m} u+\beta(-\Delta)^{2 m} v\right)\right) \\
= & \beta\left\|\nabla^{2 m+k} v\right\|^{2} \geq 0 .
\end{aligned}
$$

Therefore, $(\Lambda U, U)_{E_{k}}$ is a non-negative real number.

In order to determine the characteristic value of $\Lambda$, we consider the following characteristic equation

$$
\Lambda U=\lambda U, \forall U=(u, v)^{\mathrm{T}} \in E_{k},
$$

that is

$$
\left\{\begin{array}{l}
-v=\lambda u \\
M\left(\left\|\nabla^{m} u\right\|_{p}^{p}\right)(-\Delta)^{2 m} u+\beta(-\Delta)^{2 m} v=\lambda v
\end{array}\right.
$$

Substituting the first Equation of (4.12) into the second equation can be obtained

$$
\lambda^{2} u+M\left(\left\|\nabla^{m} u\right\|_{p}^{p}\right)(-\Delta)^{2 m} u-\beta \lambda(-\Delta)^{2 m} u=0
$$

Taking the inner product of $(-\Delta)^{k} u$, on both sides of the Equations of (4.13) respectively, we acquire

$$
\lambda^{2}\left\|\nabla^{k} u\right\|^{2}+M\left(\left\|\nabla^{m} u\right\|_{p}^{p}\right)\left\|\nabla^{2 m+k} u\right\|^{2}-\beta \lambda\left\|\nabla^{2 m+k} u\right\|^{2}=0 .
$$

Regarding (4.14) as a quadratic equation of one variable with respect to $\lambda$, for $j \in N^{+}$, and let $s=\left\|\nabla^{m} u\right\|_{p}^{p}, \quad M=M(s)$, the corresponding eigenvalues of Equation (4.11) are as follows:

$$
\lambda_{j}^{ \pm}=\frac{\beta \xi_{j} \pm \sqrt{\beta^{2} \xi_{j}^{2}-4 M\left(\left\|\nabla^{m} u\right\|_{p}^{p}\right) \xi_{j}}}{2}
$$

where $j \geq 1, \xi_{j}$ is the eigenvalue of $(-\Delta)^{2 m}$ in $H_{0}^{2 m}(\Omega)$, then $\xi_{j}=\lambda_{j} j^{\frac{2 m}{n}}$. If $\xi_{j} \geq \frac{4 M\left(\left\|\nabla^{m} u\right\|_{p}^{p}\right)}{\beta^{2}}$, then $\xi_{j} \geq \frac{4 \mu_{1}}{\beta^{2}}$, that is all the eigenvalues of $\Lambda$ are positive real numbers, and the corresponding eigenvectors are in the form of $U_{j}^{ \pm}=\left(u_{j},-\lambda_{j}^{ \pm} u_{j}\right)$. For convenience, we note that for any $j \geq 1$,

$$
\left\|\nabla^{2 m+k} u_{j}\right\|=\sqrt{\xi_{j}},\left\|\nabla^{k} u_{j}\right\|^{2}=1,\left\|\nabla^{-(2 m+k)} u_{j}\right\|=\frac{1}{\sqrt{\xi_{j}}}, k=1,2, \cdots, 2 m . \text { (16) }
$$

Theorem 4.1 Assue $\xi_{j} \geq \frac{4 M(s)}{\beta^{2}}, N_{1} \in N^{+}$is large enough, when $N>N_{1}$, the following inequality holds 


$$
\begin{aligned}
& \left(\xi_{N+1}-\xi_{N}\right)\left(\beta-\sqrt{\beta^{2} \xi_{j}-4 M(s)}-1\right) \\
& \geq 8\left(\left(C_{20} \lambda_{1}^{-m}\left(C_{21}\left\|\nabla^{2 m} \hat{u}\right\|^{\frac{\rho n}{4 m}}+C_{22}\left\|\nabla^{2 m} u\right\|^{\frac{\rho n}{4 m}}\right)+1\right)\right) .
\end{aligned}
$$

Then the operator $\Lambda$ satisfies the spectral gap condition $\Lambda_{k, 2}-\Lambda_{k, 1}>4 l_{F}$.

Proof: It is known that all the eigenvalues of $\Lambda$ are positive real numbers, $\beta \geq 2 \sqrt{\frac{M(s)}{\xi_{j}}}$, and the sequence $\left\{\lambda_{j}^{-}\right\}_{j \geq 1}$ and $\left\{\lambda_{j}^{+}\right\}_{j \geq 1}$ are monotonically increasing.

The following four steps to prove Theorem 4.1.

step 1: Because $\lambda_{j}^{ \pm}$is a non-decreasing sequence. According to Lemma 4.1, given $N$ such that $\lambda_{N}^{-}$and $\lambda_{N+1}^{-}$are consecutive adjacent eigenvalues, the eigenvalues of the operator $\Lambda$ are decomposed into $\delta_{1}$ and $\delta_{2}$, where $\delta_{1}$ is the finite parts, which are expressed as follows.

$$
\begin{gathered}
\delta_{1}=\left\{\lambda_{s}^{-}, \lambda_{j}^{+} \mid \max \left\{\lambda_{s}^{-}, \lambda_{j}^{+} \leq \lambda_{N}^{-}\right\}\right\}, \\
\delta_{2}=\left\{\lambda_{s}^{+}, \lambda_{j}^{ \pm} \mid \lambda_{s}^{-} \leq \lambda_{N}^{-} \leq \min \left\{\lambda_{s}^{+}, \lambda_{j}^{ \pm}\right\}\right\} .
\end{gathered}
$$

step 2: Consider the corresponding decomposition of $E_{k}$.

$$
\begin{aligned}
& E_{k_{1}}=\operatorname{span}\left\{U_{s}^{-}, U_{j}^{ \pm} \mid \lambda_{s}^{-}, \lambda_{j}^{+} \in \delta_{1}\right\}, \\
& E_{k_{2}}=\operatorname{span}\left\{U_{s}^{+}, U_{j}^{+} \mid \lambda_{s}^{-}, \lambda_{j}^{ \pm} \in \delta_{2}\right\} .
\end{aligned}
$$

The purpose is to make these two orthogonal subspaces of $E_{k}$ and satisfy the spectral gap Equation (4.4) is true when $\Lambda_{k, 1}=\lambda_{N}^{-}, \Lambda_{k, 2}=\lambda_{N+1}^{-}$. Further decomposition $E_{k_{2}}$, then $E_{k_{2}}=E_{k_{C}} \oplus E_{k_{R}}$,

$$
\begin{gathered}
E_{k_{C}}=\operatorname{span}\left\{U_{s}^{-} \mid \lambda_{s}^{-} \leq \lambda_{N}^{-} \leq \lambda_{s}^{+}\right\}, \\
E_{k_{R}}=\operatorname{span}\left\{U_{R}^{+} \mid \lambda_{N}^{-} \leq \lambda_{j}^{ \pm}\right\},
\end{gathered}
$$

and set $E_{k_{N}}=E_{k_{C}} \oplus E_{k_{1}}$. Note that $E_{k_{1}}$ and $E_{k_{C}}$ are finite dimensional, that $\lambda_{N}^{-} \in E_{k_{1}}, \lambda_{N+1}^{-} \in E_{k_{R}}$, and that the reason why $E_{k_{1}}$ is not orthogonal to $E_{k_{2}}$ is that, while it is orthogonal to $E_{k_{R}}$ is not orthogonal to $E_{k_{C}}$

Now we introduce two functions $\Phi: E_{k_{N}} \rightarrow R, \Psi: E_{k_{R}} \rightarrow R$, defined by

$$
\begin{aligned}
\Phi(U, V)= & 2 \beta^{2}\left(\nabla^{2 m+k} u, \nabla^{2 m+k} \bar{y}\right)+2 \beta\left(\nabla^{-(2 m+k)} \bar{g}, \nabla^{2 m+k} u\right) \\
& +2 \beta\left(\nabla^{-(2 m+k)} \bar{v}, \nabla^{2 m+k} y\right)+4\left(\nabla^{-(2 m+k)} \bar{v}, \nabla^{-(2 m+k)} g\right) \\
& -4 M\left(\left\|\nabla^{m} u\right\|_{p}^{p}\right)\left(\nabla^{k} u, \nabla^{k} \bar{y}\right), \\
\Psi(U, V)= & 2 \beta^{2}\left(\nabla^{2 m+k} u, \nabla^{2 m+k} \bar{y}\right)+\beta\left(\nabla^{-(2 m+k)} \bar{g}, \nabla^{2 m+k} u\right) \\
& +\beta\left(\nabla^{-(2 m+k)} \bar{v}, \nabla^{2 m+k} y\right)+4\left(\nabla^{-(2 m+k)} \bar{g}, \nabla^{-(2 m+k)} v\right),
\end{aligned}
$$


with $U=(u, v), V=(y, g), \bar{y}, \bar{g}$ represents the conjugate of $y$ and $g$ respectively.

For $U=(u, v) \in E_{k_{N}}$, then

$$
\begin{aligned}
\Phi(U, U)= & 2 \beta^{2}\left(\nabla^{2 m+k} u, \nabla^{2 m+k} \bar{u}\right)+2 \beta\left(\nabla^{-(2 m+k)} \bar{v}, \nabla^{2 m+k} u\right) \\
& +2 \beta\left(\nabla^{-(2 m+k)} \bar{v}, \nabla^{2 m+k} u\right)+4\left(\nabla^{-(2 m+k)} \bar{v}, \nabla^{-(2 m+k)} v\right) \\
& -4 M\left(\left\|\nabla^{m} u\right\|_{p}^{p}\right)\left(\nabla^{k} u, \nabla^{k} \bar{u}\right) \\
\geq & 2 \beta^{2}\left\|\nabla^{2 m+k} u\right\|^{2}-2\left\|\nabla^{-(2 m+k)} v\right\|^{2}-\frac{\beta^{2}}{2}\left\|\nabla^{2 m+k} u\right\|^{2}+4\left\|\nabla^{-(2 m+k)} v\right\|^{2} \\
& -4 M\left(\left\|\nabla^{m} u\right\|_{p}^{p}\right)\left\|\nabla^{k} u\right\|^{2}-2\left\|\nabla^{-(2 m+k)} v\right\|^{2}-\frac{\beta^{2}}{2}\left\|\nabla^{2 m+k} u\right\|^{2} \\
= & \beta^{2}\left\|\nabla^{2 m+k} u\right\|^{2}-4 M\left(\left\|\nabla^{m} u\right\|_{p}^{p}\right)\left\|\nabla^{k} u\right\|^{2} \\
\geq & \left(\beta^{2} \xi_{1}-4 M\left(\left\|\nabla^{m} u\right\|_{p}^{p}\right)\right)\left\|\nabla^{k} u\right\|^{2}
\end{aligned}
$$

For any $k$, there is $\beta^{2} \xi_{k} \geq 4 M\left(\xi_{k}\right)$, and according to the initial hypothesis $\mu_{0} \leq M(s) \leq \mu_{1} \leq \frac{\beta^{2} \xi_{k}}{4}$, that is $\Phi(U, U) \geq 0, \Phi$ is positive definite. Similarly, for $U=(u, v) \in E_{k_{R}}$, then

$$
\begin{aligned}
\Psi(U, U)= & 2 \beta^{2}\left(\nabla^{2 m+k} u, \nabla^{2 m+k} \bar{u}\right)+\beta\left(\nabla^{-(2 m+k)} \bar{v}, \nabla^{2 m+k} u\right) \\
& +\beta\left(\nabla^{-(2 m+k)} v, \nabla^{2 m+k} u\right)+4\left(\nabla^{-(2 m+k)} \bar{v}, \nabla^{-(2 m+k)} v\right) \\
\geq & 2 \beta^{2}\left\|\nabla^{2 m+k} u\right\|^{2}+4\left\|\nabla^{-(2 m+k)} v\right\|^{2}-\beta^{2}\left\|\nabla^{2 m+k} u\right\|^{2}-4\left\|\nabla^{-(2 m+k)} v\right\|^{2} \\
\geq & \beta^{2} \xi_{1}\left\|\nabla^{k} u\right\|^{2} .
\end{aligned}
$$

that is $\Psi(U, U) \geq 0, \Psi$ is positive definite.

Thus $\Phi$ and $\Psi$ define a scalar product, respectively on $E_{k_{N}}$ and $E_{k_{C}}$, and we can define an equivalent scalar product in $E_{k}$, by

$$
\langle\langle U, V\rangle\rangle_{E_{k}}=\Phi\left(P_{N} U, P_{N} V\right)+\Psi\left(P_{R} U, P_{R} V\right) .
$$

where $P_{N}$ and $P_{R}$ are projections of $E_{k}$ to $E_{k_{N}}$ and $E_{k_{R}}$ respectively, for brief, (4.28) can be abbreviated as the following

$$
\langle\langle U, V\rangle\rangle_{E_{k}}=\Phi(U, V)+\Psi(U, V) .
$$

We proved then to show that the subspaces $E_{k_{1}}$ and $E_{k_{2}}$ defined in (4.20), (4.21) are orthogonal with respect to the scalar product (4.28). In fact, it is sufficient to show that $E_{k_{N}}$ is orthogonal to $E_{k_{C}}$, in turn, this reduces to showing that

$$
\left\langle\left\langle U_{s}^{+}, U_{s}^{-}\right\rangle\right\rangle_{E_{k}}=\Phi\left(U_{s}^{+}, U_{s}^{-}\right)=0\left(\forall U_{s}^{+} \in E_{k_{C}}, U_{s}^{-} \in E_{k_{N}}\right) .
$$

Recalling (4.26) and (4.27), $\forall U_{s}^{+} \in E_{k_{C}}, U_{s}^{-} \in E_{k_{N}}$ 


$$
\begin{aligned}
\Phi\left(U_{s}^{+}, U_{s}^{-}\right)= & 2 \beta^{2}\left(\nabla^{2 m+k} u_{s}, \nabla^{2 m+k} \bar{u}_{s}\right)+2 \beta\left(-\lambda_{s}^{+} \nabla^{-(2 m+k)} \bar{u}_{s}, \nabla^{2 m+k} u_{s}\right) \\
& +2 \beta\left(-\lambda_{s}^{-} \nabla^{-(2 m+k)} \bar{u}_{s}, \nabla^{-(2 m+k)} u_{s}\right) \\
& +4\left(-\lambda_{s}^{+} \nabla^{-(2 m+k)} \bar{u}_{s},-\lambda_{s}^{-} \nabla^{-(2 m+k)} u_{s}\right)-4 M(s)\left(\nabla^{k} u_{s}, \nabla^{k} \bar{u}_{s}\right) \\
= & 2 \beta^{2}\left\|\nabla^{2 m+k} u_{s}\right\|^{2}-2 \beta\left(\lambda_{s}^{-}+\lambda_{s}^{+}\right)\left\|u_{s}\right\|^{2} \\
& +4 \lambda_{s}^{+} \lambda_{s}^{-}\left\|\nabla^{-(2 m+k)} u_{s}\right\|^{2}-4 M(s)\left\|\nabla^{k} u_{s}\right\|^{2} \\
= & 2 \beta^{2} \xi_{s}-2 \beta\left(\lambda_{s}^{-}+\lambda_{s}^{+}\right)+4 \lambda_{s}^{+} \lambda_{s}^{-} \frac{1}{\xi_{s}}-4 M\left(\xi_{s}\right) .
\end{aligned}
$$

according to (4.15)

$$
\lambda_{s}^{-}+\lambda_{s}^{+}=\beta \xi_{s}, \lambda_{s}^{+} \lambda_{s}^{-}=M\left(\xi_{s}\right) \xi_{s},
$$

thus, (4.30) is equivalent to

$$
\Phi\left(U_{s}^{+}, U_{s}^{-}\right)=2 \beta^{2} \xi_{s}-2 \beta\left(\lambda_{s}^{-}+\lambda_{s}^{+}\right)+4 \lambda_{s}^{+} \lambda_{s}^{-} \frac{1}{\xi_{s}}-4 M\left(\xi_{s}\right)=0 .
$$

step 3: Further, we estimate the Lipschitz constant $l_{F}$ of

$$
\begin{gathered}
F(U)=\left(0, f(x)-|u|^{\rho}\left(u_{t}+u\right)\right)^{\mathrm{T}}, \\
\forall U=(u, v)^{\mathrm{T}} \in E_{k}, U_{1}=\left(u_{1}, v_{1}\right)^{\mathrm{T}}=P_{k, 1} U, U_{2}=\left(u_{2}, v_{2}\right)^{\mathrm{T}}=P_{k, 2} U,
\end{gathered}
$$

then

$$
P_{k, 1} u=u_{1}, P_{k, 2} u=u_{2} .
$$

Give $U=(u, v), V=(\hat{u}, \hat{v}) \in E_{k}$, we get

$$
\begin{aligned}
\|F(U)-F(V)\|_{E_{k}} & =\left\|\left(0,|\hat{u}|^{\rho}\left(\hat{u}_{t}+\hat{u}\right)-|u|^{\rho}\left(u_{t}+u\right)\right)\right\| \\
& =\left\|\nabla^{k}\left(|\hat{u}|^{\rho} \hat{u}_{t}-|u|^{\rho} u_{t}\right)\right\|+\left\|\nabla^{k}\left(|\hat{u}|^{\rho} \hat{u}-|u|^{\rho} u\right)\right\| \\
& \leq C_{20}\left(\|\hat{u}\|_{\infty}^{\rho}+\|u\|_{\infty}^{\rho}\right)\left(\left\|\nabla^{k}\left(\hat{u}_{t}-u_{t}\right)\right\|+\left\|\nabla^{k}(\hat{u}-u)\right\|\right)
\end{aligned}
$$

By the interpolation inequality

$$
\begin{aligned}
& \|\hat{u}\|_{\infty}^{\rho} \leq C_{21}\left\|\nabla^{2 m} \hat{u}\right\|^{\frac{\rho n}{4 m}} \\
& \|u\|_{\infty}^{\rho} \leq C_{22}\left\|\nabla^{2 m} u\right\|^{\frac{\rho n}{4 m}}
\end{aligned}
$$

where $\rho \leq \frac{8 m}{n}$.

Therefore

$$
\begin{aligned}
& \|F(U)-F(V)\|_{E_{k}} \\
& \leq\left(C_{20} \lambda_{1}^{-m}\left(C_{21}\left\|\nabla^{2 m} \hat{u}\right\|^{\frac{\rho n}{4 m}}+C_{22}\left\|\nabla^{2 m} u\right\|^{\frac{\rho n}{4 m}}\right)+1\right)\left(\left\|\nabla^{2 m+k}\left(\hat{u}_{t}-u_{t}\right)\right\|+\left\|\nabla^{k}(\hat{u}-u)\right\|\right) \\
& \leq\left(\left(C_{20} \lambda_{1}^{-m}\left(C_{21}\left\|\nabla^{2 m} \hat{u}\right\|^{\frac{\rho n}{4 m}}+C_{22}\left\|\nabla^{2 m} u\right\|^{\frac{\rho n}{4 m}}\right)+1\right)\right)\|U-V\|_{E_{k}}
\end{aligned}
$$


thus

$$
l_{F} \leq\left(\left(C_{20} \lambda_{1}^{-m}\left(C_{21}\left\|\nabla^{2 m} \hat{u}\right\| \frac{\rho n}{4 m}+C_{22}\left\|\nabla^{2 m} u\right\|^{\frac{\rho n}{4 m}}\right)+1\right)\right)
$$

step 4: Now we need to verify that the spectral interval condition $\Lambda_{2}-\Lambda_{1}>4 l_{F}$ is established. $\Lambda_{1}=\lambda_{N}^{-}$and $\Lambda_{1}=\lambda_{N+1}^{-}$, we can get

$$
\Lambda_{2}-\Lambda_{1}=\lambda_{N+1}^{-}-\lambda_{N}^{-}=\frac{\beta}{2}\left(\xi_{N+1}-\xi_{N}\right)+\frac{1}{2}(\sqrt{R(N)}-\sqrt{R(N+1)}),
$$

with $R(N)=\beta^{2} \xi_{N}^{2}-4 M(s) \xi_{N}$.

and

$$
\lim _{N \rightarrow+\infty}\left(\sqrt{R(N)}-\sqrt{R(N+1)}+\sqrt{\beta^{2} \xi_{1}-4 M(s)}\right)\left(\xi_{N+1}-\xi_{N}\right)=0 .
$$

For formula (4.32). There, $\exists N_{1}>0$, such that for $\forall N>N_{1}$,

$$
R_{1}(N)=\frac{\beta^{2} \xi_{N}-4 M(s)}{\xi_{N}\left(\beta^{2} \xi_{1}-4 M(s)\right)}
$$

we can get

$$
\begin{gathered}
\sqrt{R(N)}-\sqrt{R(N+1)}+\sqrt{\beta^{2} \xi_{1}-4 M(s)}\left(\xi_{N+1}-\xi_{N}\right) \\
=\sqrt{\beta^{2} \xi_{1}-4 M(s)}\left(\xi_{N+1}\left(1-\sqrt{R_{1}(N+1)}\right)-\xi_{N}\left(1-\sqrt{R_{1}(N)}\right)\right), \\
\lim _{N \rightarrow+\infty} \xi_{N}\left(1-\sqrt{R_{1}(N)}\right)=0 .
\end{gathered}
$$

From the condition, it can be determined that $N_{1}>0$ such that for all $N \geq N_{1}$, and with (4.32)

$$
\begin{aligned}
\Lambda_{2}-\Lambda_{1} & =\lambda_{N+1}^{-}-\lambda_{N}^{-} \geq \frac{\xi_{N+1}-\xi_{N}}{2}\left(\beta-\sqrt{\beta^{2} \xi_{1}-4 M(s)}\right)-1 \\
& \geq 4\left(\left(C_{20} \lambda_{1}^{-m}\left(C_{21}\left\|\nabla^{2 m} \hat{u}\right\|^{\frac{\rho n}{4 m}}+C_{22}\left\|\nabla^{2 m} u\right\|^{\frac{\rho n}{4 m}}\right)+1\right)\right) \geq 4 l_{F}
\end{aligned}
$$

under the latter assumption, Theorem 4.1 is proved completely.

Theorem 4.2 In the conclusions of Theorem 4.1, initial boundary value problems admits an inertial manifold $\mu_{k}$ in $E_{k}$ of the form

$$
\mu_{k}=\operatorname{graph}(\Gamma)=\left\{\zeta_{k}+\Gamma\left(\zeta_{k}\right): \zeta_{k} \in E_{k_{1}}\right\},
$$

where $\Gamma: E_{k_{1}} \rightarrow E_{k_{2}}$ is Lipschitz continuous with the Lipschitz constant $l_{F}$,and $\operatorname{graph}(\Gamma)$ represents the diagram of $\Gamma$.

Proof: According to Theorem 4.1, Lemma 4.1 and Definition 4.1 is easily proven.

\section{Conflicts of Interest}

The authors declare no conflicts of interest regarding the publication of this paper. 


\section{References}

[1] Eden, A., Foias, C., Nicolaenko, B. and Temam, R. (1994) Exponential Attractors for Dissipative Evolution Equations. Masson Paris Wiley, New York.

[2] Dai, Z.D. and Ma, D.C. (1998) Nonlinear Wave Equation of Exponential Attractor. Chinese Science Bulletin, 43, 1269-1273. https://doi.org/10.1007/BF02883676

[3] Papadopoulos, P.G. and Stavrakakis, N.M. (2001) Global Existence and Blow-Up Results for an Equation of Kirchhoff Type on $R^{N}$. Journal of the Juliusz Schauder Center, No. 17, 91-109. https://doi.org/10.12775/TMNA.2001.006

[4] Yang, Z.J. and Wang, Y.Q. (2010) Global Attractor for the Kirchhoff Type Equation with a Strong Dissipation. Journal of Differential Equations, 249, 3258-3278. https://doi.org/10.1016/j.jde.2010.09.024

[5] Li, F.-C. (2004) Global Existence and Blow-Up of Solutions for a Higher-Order Kirchhoff-Type Equation with Nonlinear Dissipation. Applied Mathematics Letters, 17, 1409-1414. https://doi.org/10.1016/j.am1.2003.07.014

[6] Messaoudi, S.A. and Houari, B.S. (2007) A Blow-Up Result for a Higher-Order Nonlinear Kirchhoff-Type Hyperbolic Equation. Applied Mathematics Letters, 20, 866-871. https://doi.org/10.1016/j.aml.2006.08.018

[7] Shang, Y.D. and Guo, B.L. (2003) Exponential Attractor for a Class of Nonclassical Diffusion Equation. Partial Differential Equation, 16, 289-298.

[8] Eden, A., Foias, C. and Nicolaenko, B. (1994) Exponential Attractors for Dissipative Evolution Equations. Masson, Paris, 36-48.

[9] Lin, G.G., Lv, P.H. and Lou, R.J. (2017) Exponential Attractors and Inertial Manifolds for a Class of Nonlinear Generalized Kirchhoff-Boussinesq Model. Far East Journal of Mathematical Sciences, 101, 1913-1945. https://doi.org/10.17654/MS101091913

[10] Lin, G.G. and Gao, Y.L. (2017) The Global and Exponential Attractors for the Higher-Order Kirchhoff-Type Equation with Strong Linear Damping. Journal of Mathematics Research, 9, 145-167. https://doi.org/10.5539/jmr.v9n4p145

[11] Eden, A., Foias, C., Nicolaenko, B., et al. (1990) Inertial Sets for Dissipative Evolution Equations.

[12] Eden, A. (1995) Exponential Attractors for Dissipative Evolution Equations. American Mathematical Monthly, 37, 825-825.

[13] Yang, M.H. and Sun, C.Y. (2010) Exponential Attractors for the Strongly Damped Wave Equations. Nonlinear Analysis. Real World Applications, 11, 913-919. https://doi.org/10.1016/j.nonrwa.2009.01.022

[14] Dai, Z.D. and Guo, B.L. (1997) Inertial Fractal Sets for Dissipative Zakharov System. Acta Mathematicae Applicatae Sinica, 13, 279-288. https://doi.org/10.1007/BF02025883

[15] Foias, C., Sell, G.R. and Temam, R. (1988) Inertial Manifold for Nonlinear Evolutionary Equations. Journal of Differential Equations, 73, 309-353. https://doi.org/10.1016/0022-0396(88)90110-6

[16] Xu, G.G., Wang, L.B. and Lin, G.G. (2014) Inertial Manifolds for a Class of the Retarded Nonlinear Wave Equations. Mathematica Applicata, 27, 887-891.

[17] Lin, G.G. (2019) Dynamic Properties of Several Kinds of the Kirchhoff Equations. Chongqing University Press, Chongqing.

[18] Lin, G.G. (2011) Nonlinear Evolution Equation. Yunnan University Press, Kunming. 